drimer family will be obtainable, which will facilitate practical applications for TPA molecules.

YUE HU

\section{Anomalous Behavior Observed in the Magnetic State of Untwinned Lightly Doped $\mathrm{La}_{2-x} \mathrm{Sr}_{x} \mathrm{CuO}_{4}$ Single Crystals}

Researchers from the Central Research Institute of Electric Power Industry in Tokyo, Japan, have found unusual behavior in the magnetic susceptibility in detwinned $\mathrm{La}_{2-x} \mathrm{Sr}_{x} \mathrm{CuO}_{4}(x=0-0.03)$, or LSCO, single crystals. Parent insulating cuprates at high temperatures are considered to be two-dimensional (2D) Heisenberg antiferromagnets. The presence of doped holes introduces frustration into the spin system, and it has been postulated that, for the lightly doped region, the doped holes are distributed homogenously. A consequence of this homogenous picture is that once the long-range antiferromagnetic (AF) order is destroyed due to hole doping, the spin system must be isotropic. In the July 2 issue of Physical Review Letters, A.N. Lavrov, Y. Ando, S. Komiya, and I. Tsukada present a detailed study of the static magnetic susceptibility $(\chi)$ in LSCO crystals in the lightly doped region that demonstrates anisotropies in the "paramagnetic" state that are inconsistent with this picture of a 2D Heisenberg antiferromagnet. Their study of the low-temperature spin freezing in these untwinned crystals also reveals an anisotropic Curie constant and anisotropic "spin-glass" temperature.

Parallelepiped samples of LSCO single crystals $(\sim 40 \mathrm{mg})$ were prepared such that all faces coincided with the orthorhombic crystal planes to within $1^{\circ}$, and the samples were annealed in helium to remove excess oxygen. To obtain untwinned crystals, the samples were cooled slowly under a uniaxial pressure of 15-30 MPa. X-ray analysis and magnetic susceptibility measurements were performed to measure the detwinning in the bulk, and the fraction of misoriented domains was estimated to be below 5\%. The researchers used a superconducting quantum interference device (SQUID) magnetometer at fields ranging from $0.2 \mathrm{kOe}$ to $5 \mathrm{kOe}$ applied along the orthorhombic crystal axes, $a$ and $b$ ( $c$ axis normal to the $\mathrm{CuO}_{2}$ planes).

Magnetic susceptibility was measured as a function of temperature along the $a, b$, and $c$ axes in undoped $(x=0)$ AF samples. While the $\chi_{c}$ data agreed with previous studies, the $\chi_{\mathrm{a}}$ and $\chi_{\mathrm{b}}$ data displayed unexpected results, including a temperature dependence below the Néel temperature $\left(T_{\mathrm{N}}\right)$ and a deviation of $\chi_{\mathrm{a}}$ from $\chi_{\mathrm{b}}$ above $\sim 330 \mathrm{~K}$ in the "paramagnetic" state. These features reveal that suppression of the long-range Néel order in undoped LSCO does not make the system isotropic. For the lightly doped $(x=0.01)$ AF case, the researchers observed a similar deviation between $\chi_{\mathrm{a}}$ and $\chi_{\mathrm{b}}$ and no sign that an isotropic spin state was recovered above $T_{\mathrm{N}}$. Furthermore, said Lavrov, this behavior is not limited to the AF samples. Lavrov said, "We are surprised that the unexpected anisotropy persists to higher doping levels $(x=0.02,0.03)$ even after the long-range AF order is expected to be destroyed due to hole doping."

The researchers report that at low temperatures in the spin-glass state, the susceptibility is also anisotropic. Upon cooling below the spin-glass temperature, $\chi$ decreases steeply, but this freezing phenomenon is more complicated than was previously thought.

"While we are exploring several possibilities and plan to conduct further studies in order to arrive at a conclusive picture," said Lavrov, "it is clear that the magnetic state in lightly doped cuprates appears to be significantly different from a simple 2D Heisenberg antiferromagnet."

STEFFEN K. KALDOR

\section{Soft Lithography Produces Well-Aligned Carbon Nanotubes}

Carbon nanotubes' (CNTs) electronic, mechanical, and chemical properties make them potential candidates for various applications. For possible nanometerscale integrated-circuit application, it is important to develop a controlled method to synthesize high-quality CNTs. Recent research using physical masks or photo-masks have limited resolution for the resulting nanotube patterns (in micrometer scale). A team of researchers from Fudan University, China, has synthesized well-aligned CNT patterns by a soft-lithographic technique.

The support material for the catalysts is key in determining the basic parameters of CNTs, including the number of walls, the diameter, and the graphitization. As reported in the July issue of Chemistry of Materials, the researchers combined the micromolding in capillaries (MIMIC) technique with three-dimensional (3D) cubic mesoporous silica films containing iron nanoparticles as catalysts to fabricate carbon nanotube patterns with aligned orientations. The researchers incorporated the transition-metal catalysts into the ordered mesopores. The molecularly well-defined mesoporous silicon materials have large pores that allow efficient diffusion of species involved in the reactions and lead to high yield and purity of the nanotubes.
The researchers deposited well-aligned patterned CNTs on this catalyst film by chemical vapor deposition (CVD) with high yield $(400 \%)$. Scanning electron micrographs (SEMs) show that the CNTs are almost pure and also demonstrate that the distribution of the catalyst controls the growth of CNTs. Different patterns were obtained, including striplike, petal-like, and square and hexangular patterns, by using a variety of stamps.

The CNT arrays are perpendicular to the substrate. High-resolution SEM shows that the arrays are composed of thousands of CNTs compacted together. These CNTs have a length of $10 \mu \mathrm{m}$ and outer diameter of about 20-40 nm. Formations of CNT arrays are related to the pore structure. On the 3D cubicordered mesoporous silica SBA-16 with nanopores perpendicular to the substrate, perpendicular $\mathrm{CNTs}$ are obtained, while the 2D hexagonal structure SBA-15 with $1 \mathrm{D}$ channels parallel to the substrate promotes parallel growth.

Interior and wall structure information has been acquired by transmission electron microscopy of some of the typical thinner CNTs. They have a structure with multilayer graphitized carbon walls. An increase in the amount of iron catalysts leads to a large yield of CNTs, but does not modify the diameters. Finding new catalyst support materials for CNTs with smaller diameters is still the future goal.

LI ZENG

\section{Bubbles Simulate Atomic-Scale Contact at Surfaces}

Using a raft of soap bubbles to simulate the behavior of atoms, researchers at the Massachusetts Institute of Technology (MIT) have built a macroscopic system that can provide insight into the characteristics of nanoscale contact at surfaces.

In a communication published June 7 in Nature, Subra Suresh, the R.P. Simmons Professor and Head of the Department of Materials Science and Engineering at MIT and his graduate students, Andrew Gouldstone and Krystyn Van Vliet, described how they created the raft of bubbles a single-layer thick to represent an atomic layer of a material's surface. Using a high-resolution digital camera, the researchers monitored, in real time, the evolution of asperity-level contact and defect nucleation when they indented the surface from the side. They then compared data from the bubble simulation with data from nanoindentation of a variety of metals.

The experiments provide quantitative information about the location beneath the contact surface where defects nucleate 